\title{
Numerical relaxation approach for solving the general Ginzburg-Landau equations for type-II superconductors
}

\author{
Z. D. Wang \\ Texas Center for Superconductivity, University of Houston, Houston, Texas 77204 \\ C.-R. Hu \\ Center for Theoretical Physics, Department of Physics, Texas A\&M University, College Station, Texas 77843-4242
}

(Received 26 April 1991)

\begin{abstract}
A numerical relaxation approach for solving the general Ginzburg-Landau equations for type-II superconductors is developed. It is first applied to an isotropic type-II superconductor near $H_{\mathrm{cl}}$ in order to establish the reliability and effectiveness of this approach. The strength of this approach should be in dealing with anisotropic and/or inhomogeneous systems. As an initial test of this strength, we have applied it to some anisotropic cases. Distributions of the superconducting order parameter and the local magnetic field, as well as the lower critical field for these cases, are presented.
\end{abstract}

\section{INTRODUCTION}

It is well known that the Ginzburg-Landau (GL) theory provides us an important useful tool for studying the basic phenomenology of superconductivity. Since the discovery of the high-temperature superconductors, the GL theory has played an especially useful role for understanding and analyzing some physical properties of these superconductors, because there is at the present time no clear understanding of the microscopic mechanism of high- $T_{c}$ superconductors that will allow theorists to develop a microscopic theory of high- $T_{c}$ superconductors. Previously developed methods for solving the GL problem are mainly to first minimize the GL free-energy functional analytically in order to obtain the variational equations, (i.e., the GL equations,) and then to solve this resulting set of nonlinear partial differential equations analytically $^{1}$ or numerically. ${ }^{2-6}$ Unfortunately, these methods are difficult to extend to anisotropic and/or inhomogeneous systems. Since many superconductors, especially high- $T_{c}$ superconductors, exhibit strong anisotropy and/or inhomogeneity, including a difference in the directional effective masses $\left(m_{c}>m_{a} \approx m_{b}\right)$, and the effects of twin boundaries, etc., it is clearly useful to develop a general numerical scheme to handle the GL problem for such cases.

Recently, an attempt was made by Doria, Gubernatis, and Rainer to apply the simulated annealing method for such a purpose. ${ }^{7}$ In this work, we make a different attempt to treat the general GL problem based upon the relaxation method. ${ }^{8}$ This approach is to minimize the discretized GL free-energy functional directly by using numerical relaxation steps subject to the constraint of a fixed average magnetic induction $\mathbf{B}$. Compared to the simulated annealing method, the present approach should have the advantages of higher accuracy and much reduced computational work, especially for low external fields $\left(H_{\text {ext }} \ll H_{c 2}\right)$.

The relaxation approach is a direct local-optimization technique for, in general, a multivariable function. Let us consider a real function $F\left(x_{1}, \ldots, x_{n}, x_{1}^{*}, \ldots, x_{n}^{*}\right)$ of complex variables $x_{1}, x_{2}, \ldots, x_{n}$ which has a minimum at $\left(\bar{x}_{1}, \ldots, \bar{x}_{n}, \bar{x}_{1}^{*}, \ldots, \bar{x}_{n}^{*}\right)$, where $x_{i}^{*}$ is the complex conjugate of $x_{i}$. Near this minimum, one can introduce the iteration equation

$$
x_{i}^{(n+1)}=x_{i}^{(n)}-\left.\epsilon \frac{\partial F}{\partial x_{i}^{*}}\right|^{(n)},
$$

where $n$ is an integer labeling the generations of iteration and $\epsilon$ is a positive constant to be adjusted in order to optimize the convergence rate. It can be shown that $F$ will monotonically decrease to its optimum state as $n$ increases as long as we choose a proper starting state, mesh size, and the step-size parameter $\epsilon$. Consequently, we can iterate the $x_{i}$ 's until they converge to $\left(\bar{x}_{1}, \ldots, \bar{x}_{n}, \bar{x}_{1}^{*}, \ldots, \bar{x}_{n}^{*}\right)$.

The function we wish to minimize is the lattice gauge form of the GL expression for the free energy of a superconductor in the presence of a magnetic field. The variables we are concerned with are the values of the order parameter and the vector potential on a chosen mesh of points. This lattice formulation is similar to that used by Doria, Gubernatis and Rainer. ${ }^{7}$

In this paper we will illustrate the use of the relaxation approach in solving the nonlinear GL equations by first applying it to an isotropic superconductor in order to establish the approach and then extending the approach to some simple anisotropic cases. In Sec. II, we will describe the lattice gauge form of the free-energy functional, the relaxation equations for minimizing it, and the appropriate boundary conditions. In Sec. III, we will 
present the results of our calculation for the case when the external field is very close to $H_{c 1}$, assuming the system is homogeneous and isotropic and compare our results with previous results obtained by solving the radial nonlinear differential GL equations. ${ }^{6}$ In the same section, we will also present our calculations of some simple anisotropic cases with different parameter values. Finally, in Sec. IV, a short summary and conclusion will be given.

\section{LATTICE EQUATIONS AND BOUNDARY CONDITIONS}

We begin with the simple anisotropic GinzburgLandau functional for the difference $F$ between the free energies per unit volume of the superconducting state and the normal state, when choosing the magnetic field to be along the $z$ direction (which is assumed to be one of the principal directions of the system):

$$
\left.F=\left.\frac{1}{S} \int d x \int d y\left[\alpha|\psi(\mathbf{r})|^{2}+\frac{\beta}{2}|\psi(\mathbf{r})|^{4}+\sum_{k=x, y} \frac{1}{2 m_{k}}|| \frac{\hbar}{i} \nabla_{k}-\frac{2 e}{c} A_{k}\right] \psi(\mathbf{r})\right|^{2}+\frac{1}{8 \pi}[\nabla \times \mathbf{A}(\mathbf{r})]^{2}\right],
$$

where $\psi(\mathbf{r})$ is the superconducting order parameter, $\mathbf{A}$ is the vector potential, $S$ is the total area of the $x y$ plane, $m_{x}$ and $m_{y}$ are the effective masses in the $x$ and $y$ directions, respectively, and $m_{x}=m_{y}$ for the isotropic case. The variations of $F$ with respect to the fields $\psi(\mathbf{r})$ and $\mathbf{A}(\mathbf{r})$ lead to the usual differential GL equations. We will not need these equations in the following. In order to describe a superconductor in the presence of an external field, we should impose constraint on the field $\mathbf{A}$. We use the constraint of fixing the average magnetic induction $\mathbf{B}$.

To apply the numerical relaxation method, we should discretize the GL free-energy functional. ${ }^{2}$ Before doing this, we wish first to put it into a dimensionless form. Scaling energies by twice the bulk superconducting condensation energy $\alpha^{2} / \beta$ and lengths by the $x$-directional coherence length $\xi_{x}=\left(-\hbar^{2} / 2 m_{x} \alpha\right)^{1 / 2}$, setting also $\psi=\bar{\psi} \psi_{\infty}$ (where $\left.\psi_{\infty}^{2}=|\alpha| / \beta\right)$ and $\mathcal{A}=\left(2 \pi \xi_{x} / \Phi_{0}\right) \mathbf{A}$, Eq. (2) becomes

$$
F=\frac{1}{S} \int d x \int d y\left[-|\bar{\psi}|^{2}+\frac{1}{2}|\bar{\psi}|^{4}+\left|\left(\nabla_{x}-i \mathcal{A}_{x}\right) \bar{\psi}\right|^{2}+R_{m}\left|\left(\nabla_{y}-i \mathcal{A}{ }_{y}\right) \bar{\psi}\right|^{2}+\kappa_{x}^{2}(\nabla \times \mathcal{A})^{2}\right]
$$

where

$$
\kappa_{x}=\left(m_{x} c / 2 e \hbar\right) \sqrt{\beta / 2 \pi}, \quad R_{m}=m_{x} / m_{y}, \text { and } R_{m}=1
$$

for the isotropic case. Then, with the use of forward difference approximation for the derivatives and taking into account gauge invariance, we can rewrite Eq. (3) as

$$
\begin{aligned}
& F=\frac{1}{N_{x} N_{y}} \sum_{(i, j)}\left[-|\bar{\psi}(i, j)|^{2}+\frac{1}{2}|\bar{\psi}(i, j)|^{4}\right]+F_{\mathrm{kin}}+F_{\mathrm{field}}, \\
& F_{\mathrm{kin}}=\frac{1}{N_{x} N_{y}} \sum_{(i, j)}\left[\left|\bar{\psi}(i+1, j)-\bar{\psi}(i, j) e^{i d_{x} \mathcal{A}_{x}(i, j)}\right|^{2} / d_{x}^{2}+R_{m}\left|\bar{\psi}(i, j+1)-\bar{\psi}(i, j) e^{i d_{y} \mathcal{A}_{y}(i, j)}\right|^{2} / d_{y}^{2}\right], \\
& F_{\text {field }}=\frac{\kappa_{x}^{2}}{N_{x} N_{y}} \sum_{(i, j)}\left\{\left[\mathcal{A}_{x}(i, j)-\mathcal{A}_{x}(i, j+1)\right] / d_{y}+\left[\mathcal{A}_{y}(i+1, j)-\mathcal{A}_{y}(i, j)\right] / d_{x}\right\}^{2}
\end{aligned}
$$

where $N_{x}$ and $N_{y}$ are the number of lattice points in the $x$ and $y$ directions. On each lattice point ( $i, j$ ), the normalized order parameter has the value $\bar{\psi}(i, j)$, and with each point we associate horizontal and vertical bonds. The lattice constants along these bonds are $d_{x}$ and $d_{y}$, and the vector potential components on bonds $[(i, j) \rightarrow(i+1, j)]$ and $[(i, j) \rightarrow(i, j+1)]$ are denoted as $\mathcal{A}_{x}(i, j)$ and $\mathcal{A}_{y}(i, j)$, respectively. In the above lattice notation, it is easy to verify that the above expressions are invariant with respect to the gauge transformation

$$
\begin{aligned}
& \bar{\psi}(i, j) \rightarrow \bar{\psi}(i, j) e^{i \chi(i, j)}, \\
& \mathcal{A}_{x}(i, j) \rightarrow \mathcal{A}_{x}(i, j)+[\chi(i+1, j)-\chi(i, j)] / d_{x}, \\
& \mathcal{A}_{y}(i, j) \rightarrow \mathcal{A}_{y}(i, j)+[\chi(i, j+1)-\chi(i, j)] / d_{y} .
\end{aligned}
$$

Accordingly, so are $F$ and other physical quantities, such as the magnetic field and the current distribution gauge invariant.

The minimization procedure needs to be constrained to produce a given value of $B$. In practice we fix the magnitude of $B$ by specifying the total reduced flux $\Phi$ in the unit cell and implement this $B$ or $\Phi$ by imposing what will be called the "magnetic periodic boundary conditions." Let us analyze the boundary conditions. We first define the total reduced flux per unit length in the $y$ direction, $\phi\left(y_{i}\right)$ as

$$
\phi\left(y_{i}\right)=\left(2 \pi \xi_{x} / \Phi_{0}\right) \int_{o}^{L_{x}} H\left(x, y_{i}\right) d x=\oint_{\Gamma} \mathcal{A} \cdot d \mathbf{1} / \delta y_{i}
$$

where the closed path $\Gamma$ is defined in Fig. (1). This equation gives 


$$
\left[\mathcal{A}_{y}\left(L_{x}, y_{i}\right)-\mathcal{A}_{y}\left(0, y_{i}\right)\right] \delta y_{i}-\int_{0}^{L_{x}}\left[\mathcal{A}_{x}\left(x, y_{i+1}\right)-\mathcal{A}_{x}\left(x, y_{i}\right)\right] d x=\phi\left(y_{i}\right) \delta y_{i}
$$

where $L_{x} \equiv N_{x} d x, L_{y} \equiv N_{y} d y, \delta y_{i} \equiv y_{i+1}-y_{i}, \Phi_{0} \equiv h c / 2 e$ is the flux quantum, and $H(x, y) \equiv \nabla \times \mathbf{A}$ is the local magnetic field. We choose a gauge in which $\mathcal{A}_{x}$ is independent of $x$ and satisfies the following relation:

$$
\left[\mathcal{A}_{x}\left(y_{i+1}\right)-\mathcal{A}_{x}\left(y_{i}\right)\right] L_{x}=\left[\Phi / L_{y}-\phi\left(y_{i}\right)\right] \delta y_{i} .
$$

Consequently, we obtain a very simple boundary condition connecting the values of $\mathcal{A}_{y}$ at $x=0$ and $L_{x}$ :

$$
\mathcal{A}_{y}\left(L_{x}, y_{i}\right)-\mathcal{A}_{y}\left(0, y_{i}\right)=\Phi / L_{y} .
$$

Here, it is worth noting that the $\mathcal{A}_{x}$ 's cannot be fixed before optimization (to zero, for example), since they must also be regarded as independent variables. They should therefore be determined by optimization also. However, in Ref. 7, the authors chose all $\mathcal{A}_{x}\left(y_{i}\right)=0$ and also required Eq. (9), which should lead to $\phi(y)$ independent of $y$. Obviously, this is not sufficiently general, since the final results can show that $\phi(y) \propto \int H(x, y) d x$ does depend on $y$ in general. ${ }^{9}$ The present treatment avoids this problem.

The other boundary conditions need only obey gauge invariance, ${ }^{10}$ and can be taken as

$$
\begin{aligned}
& \mathcal{A}_{y}\left(x, L_{y}\right)=\mathcal{A}_{y}(x, 0) \\
& \bar{\psi}\left(x, L_{y}\right)=\bar{\psi}(x, 0) \exp (i \Phi / 2), \\
& \bar{\psi}\left(L_{x}, y\right)=\bar{\psi}(0, y) \exp \left[i y\left(\Phi / L_{y}\right)\right] .
\end{aligned}
$$

Furthermore, using Eq. (8) we can show that $\mathcal{A}_{x}(0)=\mathcal{A}_{x}\left(L_{y}\right)$, and can both be set equal to zero as part of the gauge freedom. [Note that Eq. (8) is otherwise not needed in our approach, unless one wishes to determine $\phi(y)$.]

In the following, we only consider the low-field or "isolated vortex" case, i.e., the external field $\left(H_{\text {ext }}-H_{c 1}\right) \ll H_{c 1}$ (or $\left.B \ll H_{c 1}\right)$. In this case, there is a negligible difference in energy between different choices of the lattice as long as the vortex cell is sufficiently large, so we can choose one quantum of flux (i.e., $\Phi=2 \pi$ ) in a square unit cell containing $N \times N$ lattice points.

With Eq. (4) and the above boundary conditions, we can now realize the relaxation procedure. Choosing $\bar{\psi}(i, j), \bar{\psi}^{*}(i, j), \mathcal{A}_{y}(i, j)$, and $\mathcal{A}_{x}(j)$ as independent variables, we write the relaxation iteration equations as follows:

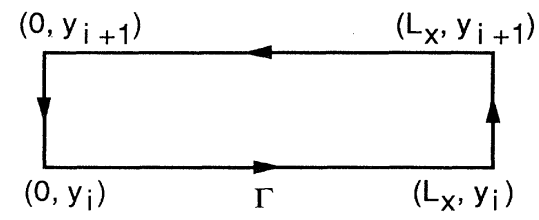

FIG. 1. The contour $\Gamma$ used in Eq. (6).

$$
\begin{aligned}
& \bar{\psi}^{(n+1)}(i, j)=\bar{\psi}^{(n)}(i, j)-\left.\epsilon_{1} \frac{\partial F_{t}}{\partial \bar{\psi}(i, j)}\right|^{(n)}, \\
& \mathcal{A}_{y}^{(n+1)}(i, j)=\mathcal{A}_{y}^{(n)}(i, j)-\left.\epsilon_{2} \frac{\partial F_{t}}{\partial \mathcal{A}_{y}(i, j)}\right|^{(n)}, \\
& \mathcal{A}_{x}^{n+1}(j)=\mathcal{A}_{x}^{(n)}(j)-\left.\epsilon_{3} \frac{\partial F_{t}}{\partial \mathcal{A}_{x}(j)}\right|^{(n)},
\end{aligned}
$$

where $\epsilon_{1}, \epsilon_{2}$, and $\epsilon_{3}$ are all positive numbers, $F_{t}=N_{x} N_{y} F$.

\section{RESULTS}

In our numerical calculation, the parameters chosen are (1) $N_{x}=N_{y}=100$ (for $\kappa=10, N_{x}=N_{y}=200$ ) and (2) the lattice constants $d_{x}=d_{y}=0.4 \xi_{x}\left(d_{x}=d_{y}=0.2 \xi_{x}\right.$ was also chosen for $\kappa=1$, and we found no obvious differences in our calculated results for $H_{c 1}$ between these two cases). These choices ensure that our calculation satisfies the condition $B<H_{c 1}$. The relaxation step-size parameters $\left(\epsilon_{1}, \epsilon_{2}, \epsilon_{3}\right)$ are chosen to range from 0.0007 to 0.01 , and they are adjusted in order to ensure convergence of iteration and fast iteration speed. We iterate Eq. (11) until the relations $\left\langle\partial F_{t} / \partial x_{i}^{(l)}\right\rangle\left\langle\bar{\epsilon}\right.$ and $\max \left(\partial F_{t} / \partial x_{i}^{(l)}\right)<\epsilon_{M}$ are satisfied. Here $\langle\cdots\rangle$ represents taking the average over
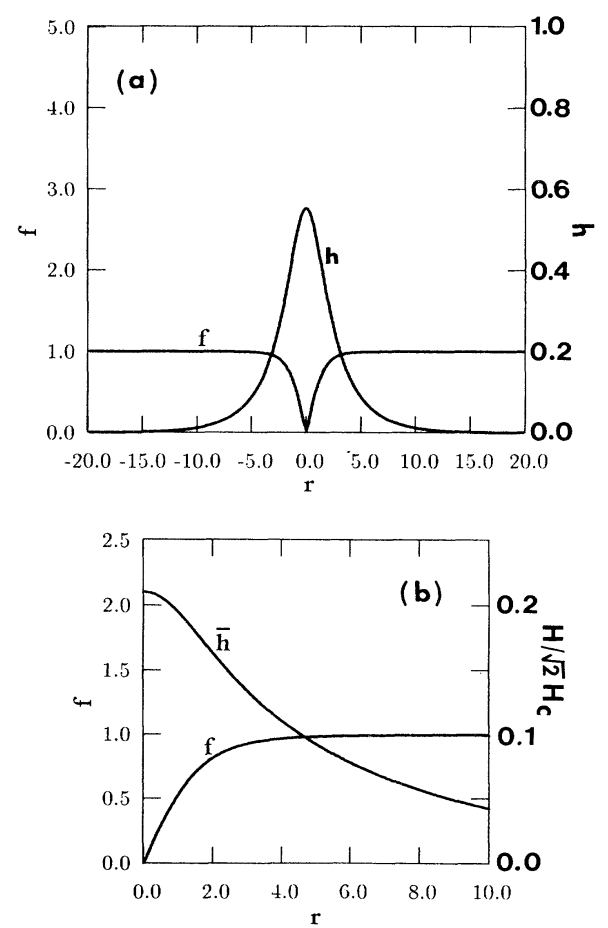

FIG. 2. (a) At $R_{m}=1.0$ and $\kappa_{x}=3.0$, the distribution of the magnitude of the order parameter $f$ and the reduced local magnetic field $h \equiv H / H_{c} ;\left(\right.$ b) at $R_{m}=1.0$ and $\kappa_{x}=10.0$, the distributions of $f$ and $\bar{h} \equiv h / \sqrt{2}$. 
the lattice points, and $x^{(l)}(l=1,2,3)$ correspond to $\bar{\psi}$, $\mathcal{A}_{y}, \mathcal{A}_{x}$. (We choose $\bar{\epsilon} \sim 10^{-5}$, and $\epsilon_{M} \sim 10^{-4}$ in most cases.) The distributions of the magnitude of the order parameter $f(\equiv|\bar{\psi}|)$ and the reduced local field $h \equiv H / H_{c}$ (or $\bar{h} \equiv h / \sqrt{2}$ ) are obtained with higher accuracy and are plotted in Figs. 2(a) and 2(b). If we overlap the plots of $f$ and $\bar{h}$ for $\kappa=10$ obtained by Matricon ${ }^{11}$ on top of the curves in Fig. 2(b), we find the two sets of curves practically coincide.

From the distributions of $\bar{\psi}$ and $H$, we can calculate the external magnetic field $H_{\text {ext }}$, which approaches the lower critical field $H_{c 1}$ as $B \rightarrow 0$. We compute $H_{\text {ext }}$ based on the discretized form of the virial relation ${ }^{10}$

$$
H_{\mathrm{ext}} B=H_{c}^{2}\left(F_{\mathrm{kin}}+2 F_{\text {field }}\right),
$$

where $H_{c} \equiv\left(4 \pi \alpha^{2} / \beta\right)^{1 / 2}$ is the thermodynamic critical field. ${ }^{12}$ For the isotropic case and $B \rightarrow 0$, it can be shown that the above equation is equivalent to Eq. (14) of Ref. 13. The values of the lower critical field $H_{c 1}$ for different values of $\kappa$ are listed in Table I, and are compared with the results obtained by Neumann and Tewordt by directly solving the nonlinear radial differential GL equations. ${ }^{6}$ We can see that within three significant digits our results are in agreement with those obtained in Ref. 6. This implies that our method can have high accuracy. This also demonstrates that the relaxation approach developed here is reliable and effective for solving the general GL problem.

Next, to demonstrate that the present approach has the potential to extend beyond the previous approaches to anisotropic and/or inhomogeneous systems, we use this relaxation approach to investigate a simple anisotropic GL problem (with $\mathbf{H} \| \widehat{\mathbf{z}}$, and $m_{x} \neq m_{y}$ ). As mentioned in Sec. II, when $B \rightarrow 0$, we can choose the same square unit cell as that used in the isotropic case, and also the same boundary conditions. The distributions of $(h(x, 0), h(0, y))$ and $(f(x, 0), f(0, y))$ for several anisotropic cases are shown in Fig. 3, and some are contour plotted in Fig. 4. We can find obvious anisotropy effects on $f$ and $h$. The shapes of the vortex cores and the field distributions are clearly deformed as expected qualitatively. We have also calculated $H_{c 1}$ for several different values of the anisotropy parameter $R_{m} \cdot H_{c 1}$ as a function of $\kappa$ and $R_{m}$ (or $1 / R_{m}$ ) is shown in Fig. 5 and Figs. 6(a) and $6(b)$.

From Fig. 5 (or Fig. 6), we can conclude that, for anisotropic superconductors with $m_{a}=m_{b}<m_{c}$, or $\xi_{a, b}>\xi_{c}$, (a,b,c are the three principal directions), the lower critical field in the $c$ direction (corresponding to $R_{m}=1$ ) is larger than those in the directions that lie in the $a b$ plane (corresponding to $R_{m}<1$ ). This is consistent with measurements on all anisotropic superconductors including high- $T_{c}$ superconductors. From our numerical results presented in Fig. 5, we also find that $H_{c 1} / H_{c}$ actually depends only on $\kappa_{x}^{2} / R_{m}\left(\equiv \bar{\kappa}^{2}\right)$. This is consistent with the conclusion of an earlier work by Klemm and Clem, ${ }^{14}$ who showed that the free-energy functional of an anisotropic superconductor can always be brought into the form of that of an isotropic superconductor by an ordered sequence of three transformations:
TABLE I. Comparison of calculated values of $H_{c 1} / H_{c}$ between this work and Ref. 6.

\begin{tabular}{ccc}
\hline \hline$\kappa$ & This work & Ref. 6 \\
\hline 1.0 & 0.8182 & 0.818 \\
2.0 & 0.5493 & 0.549 \\
3.0 & 0.4330 & 0.433 \\
4.0 & 0.3664 & \\
5.0 & 0.3174 & 0.317 \\
10.0 & 0.2020 & 0.202 \\
\hline \hline
\end{tabular}
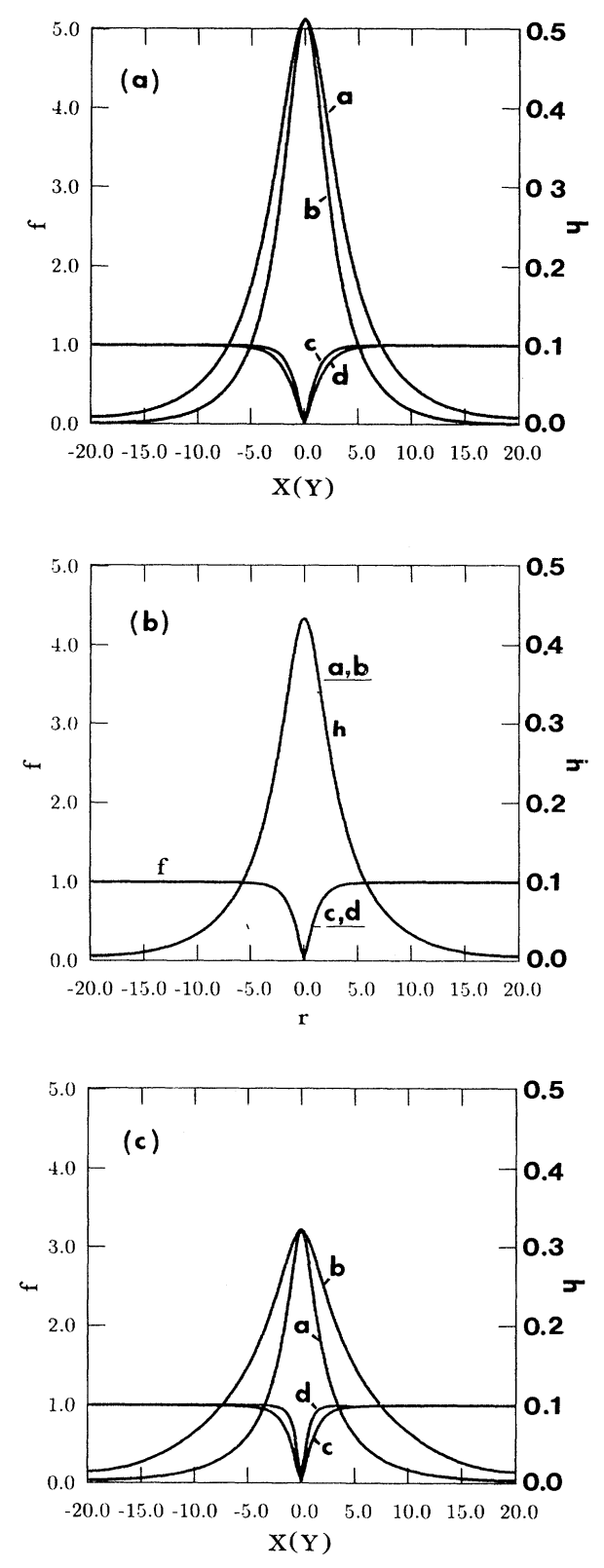

FIG. 3. At $\kappa_{x}=5.0$, the distributions of a: $h(0, y) ; \mathrm{b}: h(x, 0)$; c: $f(x, 0)$; and d: $f(0, y)$ for (a) $R_{m}=2.0$, (b) $R_{m}=1.0$, and (c) $R_{m}=0.25$. 
an anisotropic scale transformation, followed by a rotation, which is followed by another simple scale transformation. In this case, when the applied field is along one of the principle directions (i.e., along $\widehat{\mathbf{e}}_{3}$ of Ref. 14, which is the $z$ direction here), this sequence of transformation reduces to a simple anisotropic scale transformation, viz.,

$$
\begin{aligned}
& (x, y)=\left(R_{m}^{-1 / 4} \bar{x}, R_{m}^{1 / 4} y\right), \\
& (\partial x, \partial y)=\left(R_{m}^{1 / 4} \partial_{x}, R_{m}^{-1 / 4} \partial_{y}\right),
\end{aligned}
$$

and
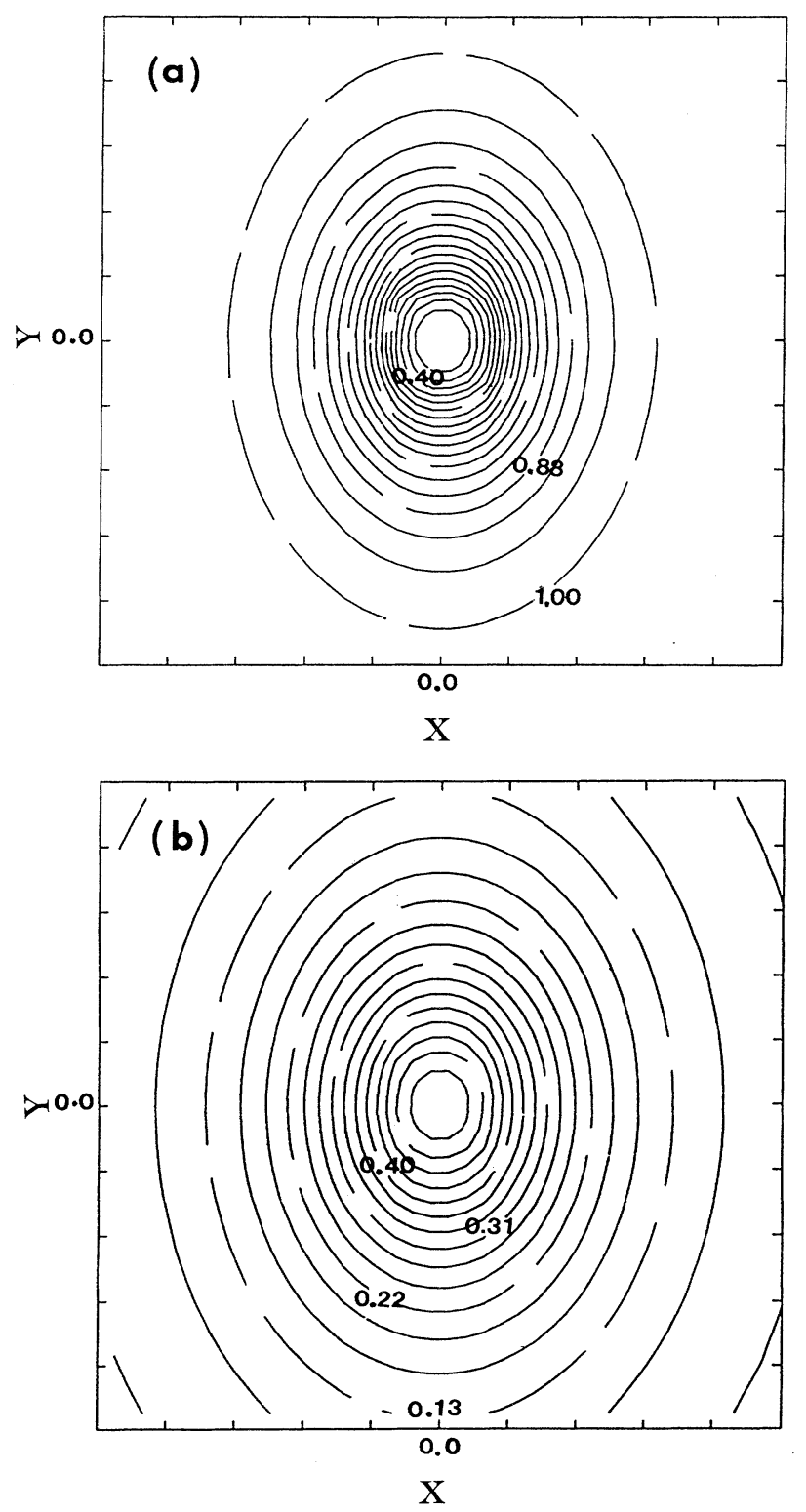

FIG. 4. At $\kappa_{x}=5$ and $R_{m}=2.0$, (a) constant $f$ contours with the increment of $f$ between neighboring contours being 0.04 . The $x$ and $y$ intervals are 1.04; (b) Constant- $h$ contours with the increment of $h$ between neighboring contours being 0.03 . The $x$ and $y$ intervals are 1.56 .

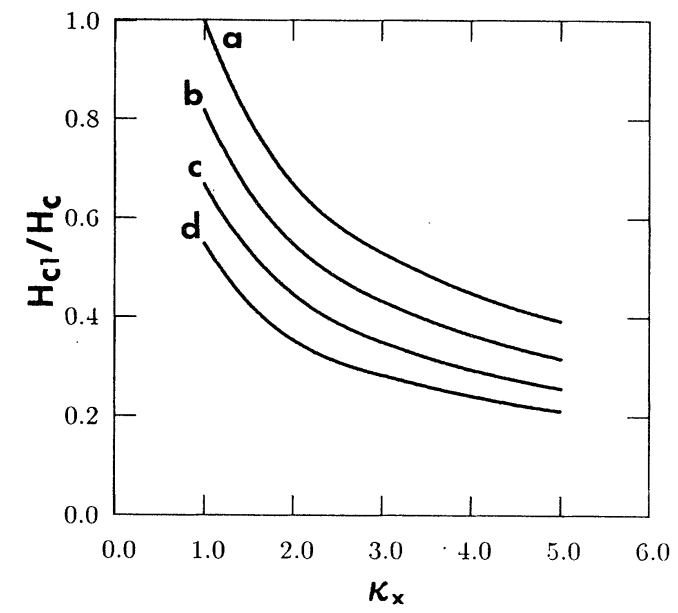

FIG. 5. $H_{c 1} / H_{c}$ as a function of $\kappa_{x}$ for different anisotropyparameter values. a: $R_{m}=2.0$; b: $R_{m}=1.0$; $: R_{m}=0.5$; and d: $R_{m}=0.25$.
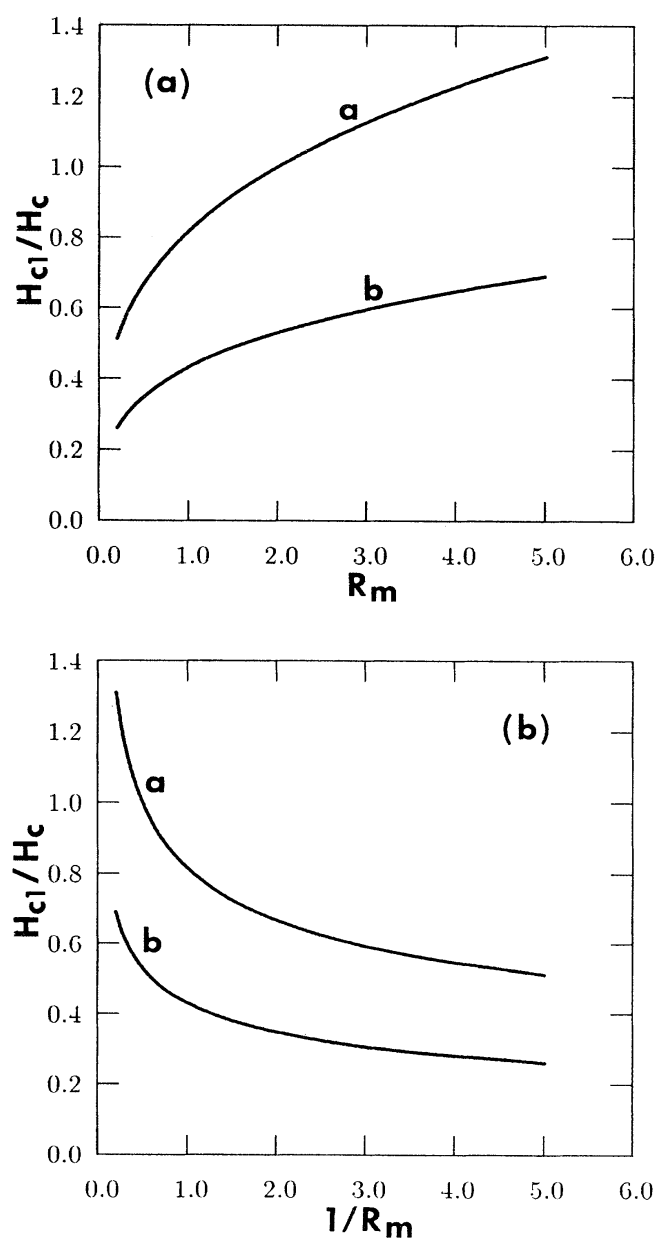

FIG. 6. (a) $H_{c 1} / H_{c}$ as a function of $R_{m}$ for fixed $\kappa_{x}$. a: $\kappa_{x}=1.0$; b: $\kappa_{x}=3.0$. (b) $H_{c 1} / H_{c}$ as a function of $1 / R_{m}$ for fixed $\kappa_{x}$. a: $\kappa_{x}=1.0 ; \mathrm{b}: \kappa_{x}=3.0$. 


$$
\left(A_{x}, A_{y}\right)=\left(R_{m}^{1 / 4} \bar{A}_{\bar{x}}, R_{m}^{-1 / 4} \bar{A}_{\bar{y}}\right),
$$

which leaves $\psi$ and $\mathbf{H}=\boldsymbol{\nabla} \times \mathbf{A}$ invariant. It also converts the third term of Eq. (2) to the isotropic form

$$
\frac{1}{2 \bar{m}} \sum_{\bar{k}=\bar{x}, \bar{y}}\left|\left(\frac{\hbar}{i} \nabla_{\bar{k}}-\frac{2 e}{c} \bar{A}_{\bar{k}}\right) \psi(\overline{\mathbf{r}})\right|^{2}
$$

with $\bar{m} \equiv \sqrt{m_{x} m_{y}}$, which clearly implies that $H_{c 1}\left(m_{x}, m_{y}\right)=H_{c 1}(\bar{m}, \bar{m})$, or $H_{c 1}\left(\kappa_{x}, R_{m}\right)$ depends only on $\kappa_{x} / \sqrt{R_{m}} \equiv \bar{\kappa}$. Locally it also implies that

$$
\begin{aligned}
& \psi\left(m_{x}, m_{y}, x, y\right)=\psi\left(\bar{m}, \bar{m}, R_{m}^{1 / 4} x, R_{m}^{-1 / 4} y\right), \\
& H\left(m_{x}, m_{y}, x, y\right)=H\left(\bar{m}, \bar{m}, R_{m}^{1 / 4} x, R_{m}^{-1 / 4} y\right) .
\end{aligned}
$$

In fact, this transformation implies a lot more than these results at $H_{\text {ext }}=H_{c 1}$, since it is valid for all fields between $H_{c 1}$ and $H_{c 2}$, as long as the applied field is along a principal direction (called $z$ ). In fact, it implies that all thermodynamic quantities in this case depend only on $\bar{\kappa}$, and that Eq. (13) is valid at all $H_{\text {ext }}$. It also implies that numerical solution of the static GL equations of an anisotropic superconductor is actually not needed when the applied field is along a principal direction. ${ }^{15}$ It is done here only for the purpose of establishing the method so it can be used for studying more complex situations, such as where there are twinning boundaries present, or when the anisotropy of the system cannot be described by the anisotropic mass model.

\section{SUMMARY AND CONCLUSION}

We have presented and demonstrated the utility of a relaxation approach to the solution of the general GL problem. Our main intent here is to make sure that the relaxation approach works for this purpose and also to set up the program. We believe we have achieved this goal. Meanwhile, we have obtained accurate numerical results for some simple anisotropic superconducting vortex states. Our future objectives are to study complicated anisotropic or inhomogeneous superconducting states quantitatively using this approach, including the case when twin boundaries exist, which is important for, for example, the high- $T_{c}$ superconductor $\mathrm{Y}-\mathrm{Ba}-\mathrm{Cu}-\mathrm{O}$. In addition, we can calculate the $\boldsymbol{M}-\boldsymbol{H}$ curves for anisotropic superconducting states, and determine the structure of the vortex lattice for these cases. Although the computational work will be substantially more than that in the present work, it is still expected to be much less than if the simulated annealing method is used for the same purposes. ${ }^{6}$ Therefore, we believe that the relaxation approach can play an important role in studying the general GL problem under complicated situations, including the present of anisotropy and/or inhomogeneity.

\section{ACKNOWLEDGMENTS}

We are grateful to Professor C. S. Ting for his helpful discussions. We also wish to thank Mr. Jin Yu for his assistance in the computational work. This work is supported by a grant from the Texas Center for Superconductivity at the University of Houston funded under the prime Grant No. MDA-972-88-G-0002 from the Defense Advanced Research Project Agency and the state of Texas.
${ }^{1}$ See, for example, Superconductivity, edited by R. D. Parks (Marcel Dekker, New York, 1969); and D. Saint-James, E. J. Thomas, and G. Sarma, Type-II Superconductivity (Pergamon, Oxford, 1969).

${ }^{2}$ A. A. Abrikosov, Zh. Eksp. Teor. Fiz. 32, 1442 (1957) [Sov. Phys. JETP 5, 1174 (1957)].

${ }^{3}$ W. M. Kleiner, L. M. Roth, and S. H. Autler, Phys. Rev. 133, A1225 (1964).

${ }^{4}$ E. H. Brant, Phys. Status Solidi B 51, 345 (1972).

5 J. L. Harden and V. Arp, Cryogenics 3, 105 (1963).

${ }^{6}$ L. Neumann and L. Tewordt, Z. Phys. 189, 55 (1966).

${ }^{7}$ M. M. Doria, J. E. Gubernatis, and D. Rainer, Phys. Rev. B 41, 6335 (1990).

${ }^{8}$ S. L. Alder and T. Piram, Rev. Mod. Phys. 56, 1 (1984).

${ }^{9}$ For $H_{\text {ext }} \simeq H_{c 2}, \phi(y)$ is practically a constant. For $H_{\text {ext }} \simeq H_{c 1}$, $\phi(y)$ is nearly zero everywhere. Thus in both of these limits, the error caused by approximating $\phi(y)$ by a constant will be small. However, for the intermediate field range $H_{c 1}<H_{\text {ext }}<H_{c 2}$, (or $0<B<H_{c 2}$ ), we expect the error not to be negligible, but we have not yet systematically analyzed how large a maximum percentage error it can cause.

${ }^{10}$ M. M. Doria, J. E. Gubernatis, and D. Rainer, Phys. Rev. B
39, 9573 (1989).

${ }^{11}$ See Superconductivity (Ref. 1), Chap. 14, Fig. 11. In that figure one should notice a slight error in the vertical scale, viz., the 0.20 mark is somewhat misplaced.

${ }^{12}$ At first sight, it would appear that Eq. (12) gives $H_{c 1}$ as the ratio of two very small quantities, and therefore it might have very poor accuracy for this purpose. Actually, this is not so. This is best seen by multiplying both sides of the equation by the area of the unit cell, which converts the factor $B$ on the left-hand side to a unit quantum of flux, and the whole righthand side to also a finite quantity.

${ }^{13}$ C. -R. Hu, Phys. Rev. B 6, 1757 (1972).

${ }^{14}$ R. A. Klemm and J. R. Clem, Phys. Rev. B 21, 1868 (1980).

${ }^{15} \mathrm{We}$ mention in passing that when the applied field is not along one of the principal directions the predictions of the anisotropic-mass GL model becomes far more complex and have been the subject of extensive past and recent studies. See, besides Ref. 14 above, also V. G. Kogan and J. R. Clem, Phys. Rev. B 24, 2497 (1981); K. G. Petzinger and G. A. Warren, ibid. 43, 2023 (1990); Z. Hao et al., ibid. 43, 2844 (1991); Z. Hao and J. R. Clem, ibid. 43, 7622 (1991); and (unpublished). 